\title{
Use of information technology and education improved screening for cognitive impairment in the over 65s
}

\author{
Authors: Clare Coyle, Josephine Prynn, Sarah Kingdon
}

\section{Aims}

This project aimed to improve identification and management of older patients with cognitive impairment on hospital admission.

\section{Methods}

All medical patients over 65 should have AMTS on admission and AMTS $<8$ should trigger further investigation. All medical admissions aged over 65 over a 2-week period in 2015 were identified. The electronic clerking was used to identify whether AMTS had been documented and, if less than 8 , whether there had been appropriate investigation and referral. Comparison was made to a previous audit in 2012 when paper clerking proformas had been used, which included a prompt for AMTS.

Subsequently, a new clerking proforma for over $65 \mathrm{~s}$ was designed using Cerner CRS with a pre-populated AMTS. Clerking doctors were trained on the rationale behind early diagnosis of cognitive impairment and on the new proforma. Reminders were placed on all computers used for clerking. The data were then re-audited.

\section{Results}

The proportion of eligible patients with AMTS on admission was initially lower in $2015 ; 27 / 184$ (14.7\%) compared to 2012 ; 26/114 (22.8\%). This improved following intervention: 42/169 (24.8\%). Of those patients with AMTS <8, 1/23 (4.3\%) had appropriate investigations, compared to $2012,11 / 15$ (73.3\%); $1 / 23(4.3 \%)$ was referred to the psychiatric team. Following intervention, $12 / 24(50 \%)$ had appropriate investigations and $4 / 24(16.7 \%)$ were referred to psychiatry.

The over 65 s proforma was used for $37 / 169$ (21.9\%) patients following its introduction, of which 18/37 (48.6\%) included an AMTS. When the standard clerking proforma was used, 18/142 (18.2\%) clerkings included an AMTS.

\section{Conclusions}

Cognition screening in the over 65 s on admission to our hospital is poor, as is the onward investigation and referral of those identified. The use of the pre-populated clerking proforma alongside extensive teaching of clerking doctors successfully improved the number screened in this population, leading to better rates of appropriate investigation and referral to psychiatric services. However, we are still far from the goal of $100 \%$ uptake. A cultural change needs to occur in order to instil the importance of early detection of dementia, and as such we will aim to include training on cognitive impairment screening at trust induction. Improvement in this area will allow earlier diagnosis of dementia, and is an integral part of improving patient care. This study also illustrates a valid way to use information technology to improve uptake of a new intervention alongside education and teaching. There is scope to develop the proforma to tailor it further to older patients' care needs. 